\title{
New Dining Technology Based on Virtual Reality
}

\author{
${ }^{1}$ Reni Hena Helan R, ${ }^{2}$ Kumudavalli R, ${ }^{3}$ Subhasri S A, ${ }^{4}$ Aishwarya G \\ ${ }^{1}$ Assistant Professor, Department of Computer Science and Engineering, Dhanalakshmi College Of Engineering, \\ Chennai, Tamilnadu, India \\ ${ }^{2,3,4}$ U.G Student, Department of Computer Science and Engineering, Dhanalakshmi College Of Engineering, Chennai, \\ Tamilnadu, India
}

\begin{abstract}
In this paper, we enclose that the simplicity and ease of accessing a menu are the main aspects that facilitate food ordering system in a restaurant. Holographic technology can completely record and reconstruct the information of object. Therefore, the holographic display can provide important role for various fields like Medical Treatment, Industry, Entertainment and other field. Hence, we use hologram in dining system of restaurant. A Hologram menu completely revolutionizes the patron's dining experience. Existing system provide a manual based menu card. This paper aims to provide a solution using a recommendation algorithm that recommends dishes on a tablet menu in a restaurant. In addition to this, the amount for the menu items ordered will also be displayed in the $L C D$ and the total bill amount will be sent to the mobile numbers of the customers.
\end{abstract}

Keywords: Hologram, GSM, Zigbee, Liquid Crystal Display (LCD), UART

\section{INTRODUCTION}

The recorded interference pattern between a point sourced of light of fixed wavelength and a wave field scattered from the object is a hologram. A hologram is recorded in a two- or three-dimensional medium and contains information about the entire three-dimensional wave field of the recorded object. When the hologram is illuminated by the reference beam, the diffraction pattern recreates the light field of the original object. The viewer is then able to see an image that is indistinguishable from the recorded object. In a recording media (e.g. a CD) the grooves contain information about sound that can be used for song reconstruction and a holographic plate contains information about light that is used to reconstruct an object.

Holograph is a photographic technique that presents a 3D image by recording light scattered from an object. Holography is an eye catching imaging technique as it gives a whole three-dimensional view of a single image. This technique generates object's representations that are real look alike 3D renditions since it records the details in such a way they are closer to what the eyes of people would see in the world.

As years passed, there were many holographic techniques that came into existence. A few such techniques included are transmission holograms, that allow light to be shined through them and the image to be viewed from the side, and rainbow holograms.

\section{LITERATURE SURVEY}

In the paper ${ }^{[11]}$, titled "Surface Susceptibility Synthesis of Meta surface Holograms for Creating Electromagnetic Illusions" Tom J. Smy, Scott A. Stewart, Shulabh Gupta, proposed a general design method for synthetic surface holograms based on integral equation (IE) and the Generalized Sheet Transition Condition (GSTC), in which the meta surface hologram is described as a sheet with zero thickness with surface susceptibility densities. Several selected configurations were chosen to illustrate various aspects of creating a two-dimensional hologram, as well as a new numerical method for artificially re-propagating the scattered field required for the synthesis process. Finally, the influence of the size of the meta surface and the intensity of the illumination field on the quality of the reconstructed scattered field is discussed.

Yang Wu, Jun Wang, Chun Chen, Chan-Juan Liu in the paper ${ }^{[12]}$, titled "Generation of Phase-Only Fourier Hologram Based on Double Phase Method and Quantization Error Analysis" proposed that the double phase technique is a higher 
manner for generating phase-most effective holograms of excessive reconstruction great due to no addition of a random phase. However, it can not at once encode the Fourier spectrum due to the constrained gray modulation variety of spatial light modulator, and quantization error. This scarcity is proscribing the double phase method in the phase-most effective Fourier hologram, and this problem is in no way discussed, solved as far as our knowledge is concerned. To resolve this problem, Yang Wu et. Al. proposed a technique to generate phase-most effective Fourier hologram with the aid of using reading quantization error, and including a right quadratic phase.

The paper ${ }^{[13]}$, titled Hologram Domain Data Compression: Performance of Standard Codecs and Image Quality Assessment at Different Distances and Perspectives by Roberto Corda et. Al. proposed a goal assessment of the performances of state-of-the-art compression strategies carried out to virtual holographic information. The experimental evaluation takes into consideration numerous holographic images reconstructing the holograms from specific views and distances. The outcomes of the exploratory observe are reported, mentioned and complemented with a statistical evaluation of the hologram information below test, offering insights for computer-generated holography compression strategies design.

Enhancement of Depth Range in LED-based Holographic Near-Eye Display Using Focus Tunable Device ${ }^{[14]}$, by Jaebum Cho et. Al. defined that the Light emitting diode (LED) has been a distinguished factor for illumination supply of holographic presentations. It can replace laser in that it alleviates speckle noise in reconstructed hologram and is appeared as a safer supply. However, LED-primarily based totally holographic presentations can reconstruct an photo in a restrained intensity variety because of in part coherent function of LED itself. In this paper, a prototype of LEDprimarily based totally holographic near-eye display with attention tunable lens to extend the depth range. The feasibility of the system is supported with the aid of using numerous experimental results.

Jonas Schild in Applying Multi-User Virtual Reality to Collaborative Medical Training [15], presented a multi-user virtual reality (VR) setup that aims at providing novel training tools for paramedics presented a multi-consumer virtual reality (VR) setup that targets at presenting novel training tools for paramedics that complements present learning methods. The prototype gives hands-on experience on multi-consumer VR in an carried out scenario, generating discussion round current state and future improvement regarding three essential studies areas: (a) consumer navigation, (b) interaction, (c) stage of visible abstraction, and (d) stage of project abstraction.

\section{SYSTEM DESIGN}

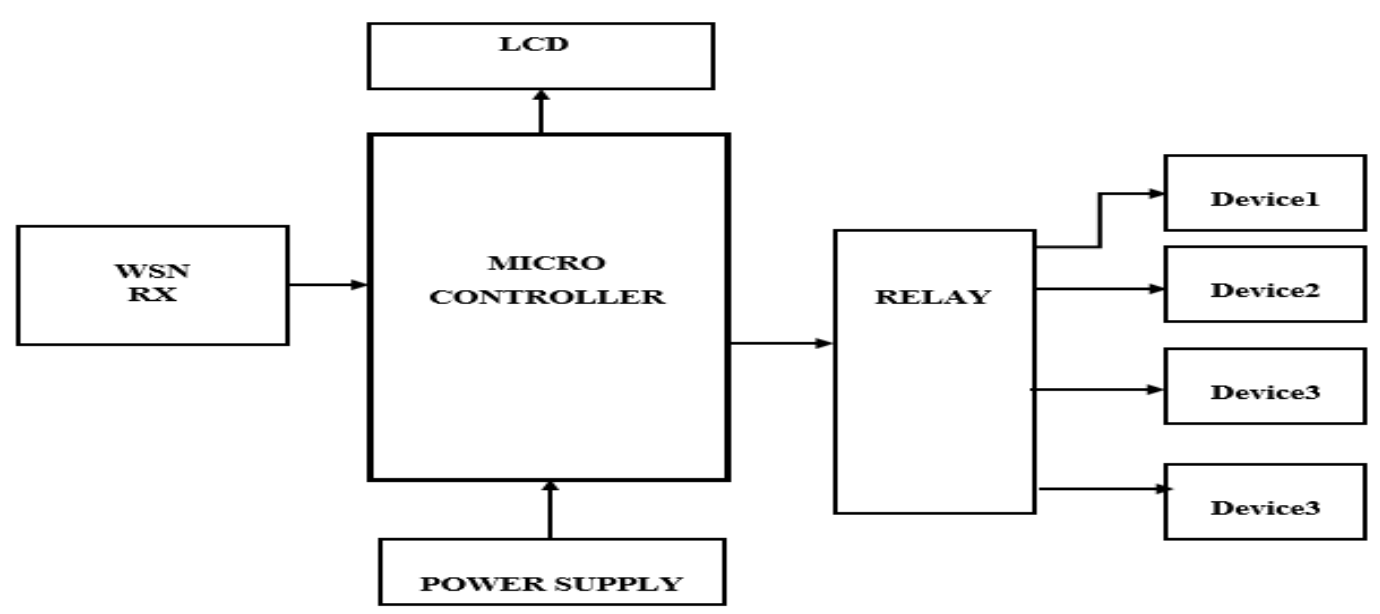

Fig. 3.1 System Design

In hologram, the detection of wavelength distribution of a light source happens by the use of a multiplex Fresnel hologram. In order to measure the wavelength distribution of the light source, a spectrometer is typically used, however in this example it's miles hard to measure the wavelength distribution even as the use of the light source. To separate the block in light source food, fabric etc., we use Transmission and receiver section. Fig 3.1 shows a clear view of the system design. 


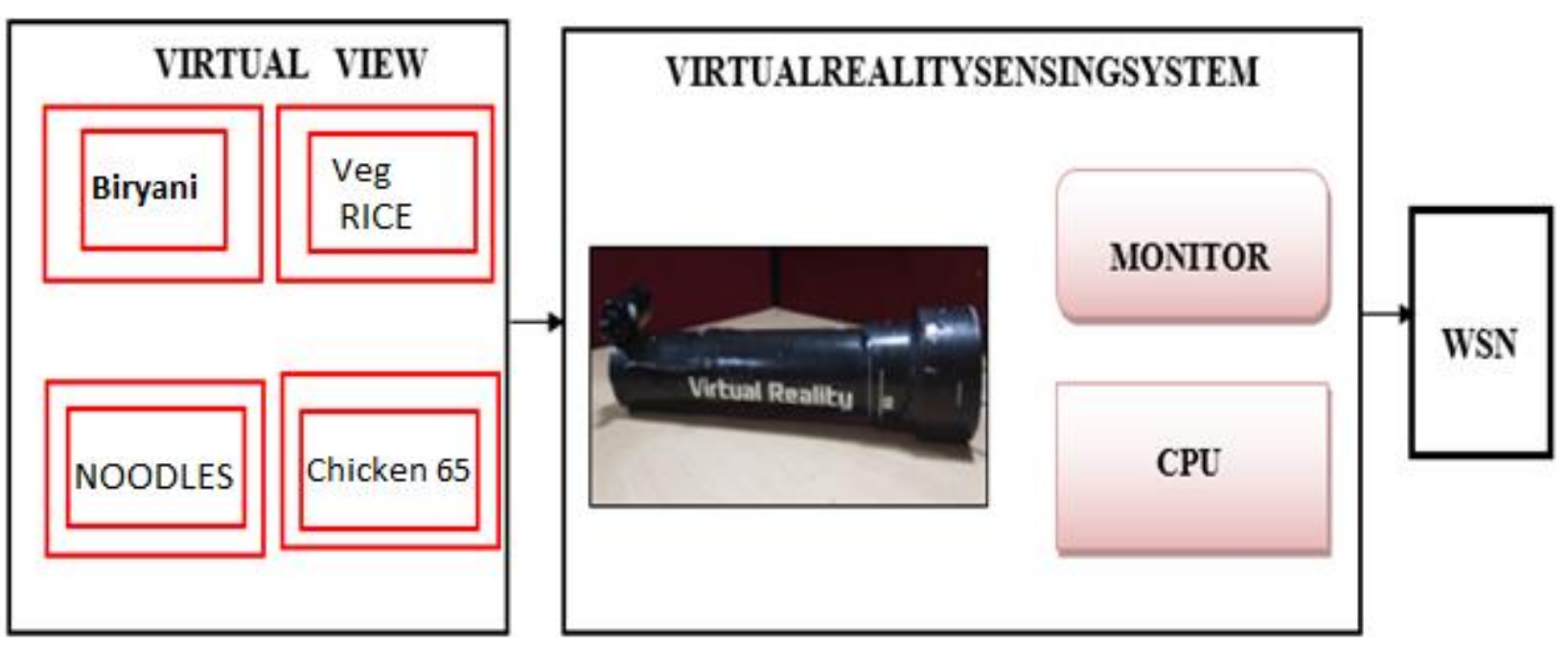

Fig. 3.2 Architecture Diagram

This Architecture diagram shown in Fig 3.2 describes a virtual view for the menu being displayed with Virtual reality sensing system the usage of Hologram torch, Monitor, CPU. These are connected to the WSN. The menu are displayed at the display screen virtually by the usage of a Hologram torch which allows the person to choose a menu object based totally on their interest. The menu decided on by the customer can be exhibited to the person through the usage of a LCD Display unit and the price for all the chosen objects can also be displayed. Once the customer finishes the ordering of the menu objects, the entire invoice for the order can be despatched to the consumer's cell through Global System for Mobile Communications (GSM) to make the payment.

\section{COMPONENTS USED}

\section{A.TORCH LIGHT (TRANSMISSION LIGHT)}

The photographic approach of recording light is scattered from an object and imparting it as 3-D picture is referred to as as holography. The object illustration generated through this approach life-like 3-D renditions as it stores data in a way in the direction of anything our eyes used to look in the world around us.

\section{B.ARDUINO UNO (MICROCONTROLLER)}

Arduino is a tool for making computer systems which can experience and manage greater of the physical world than your desktop computer. It is an open-source physical computing platform based on a easy microcontroller board, and a development environment for writing application for the board. Arduino may be used to create interactive objects, taking inputs from plenty of switches or sensors, and controlling a variety of lights, motors, and different physical outputs. Arduino initiatives may be stand-alone, or they may be communicate with application working on the computer (e.g. Flash, Processing, MaxMSP).

\section{C.LCD DISPLAY}

The 16x2 LCD used on this experiment has a total of sixteen pins. Eight of the pins are data lines (pins 7-14), two are for power and ground (pins 1 and 16), three are used to govern the operation of LCD (pins 4-6), and one is used to alter the LCD display screen brightness (pin 3). The last pins (15 and 16) power the backlight.

\section{D.RELAY}

A relay is an electrically operated switch. Current flowing via the coil of the relay creates a magnetic field which draws a lever and modifies the transfer contacts. The coil current may be on or off so relays have switch positions and they are double throw (changeover) switches. Relays permit one circuit to switch a second circuit which may be absolutely separate from the first. For instance a low voltage battery circuit can use a relay to transfer a $230 \mathrm{~V}$ AC mains circuit. 


\section{E.TRANSFORMER}

A transformer is a static piece of which electric powered energy in a single circuit is converted into electric powered energy of equal frequency in some other circuit. It can increase or decrease the voltage in the circuit, however with a corresponding reduction or rise in current. It works with the precept of mutual induction. In our project we use a step down transformer for offering a important supply for the digital circuits. Here we step down a 230volts ac into 12volts ac.

\section{F.RECTIFIER}

A dc level received from a sinusoidal input may be progressed $100 \%$ the use of a procedure called full wave rectification. Here in our venture for full wave rectification we use bridge rectifier. From the simple bridge configuration, we see that diodes (say D2 \& D3) are conducting at the same time as the alternative diodes (D1 \& D4) are in off state all through the duration $t=$ zero to $T / 2$. Accordingly for the negative cycle of the input the conducting diodes are D1 \& D4. Thus the polarity across the load is the same.

\section{G.FILTERS}

In order to attain a dc voltage of $0 \mathrm{~Hz}$, we should use a low pass filter. So that a capacitive filter circuit is used wherein a capacitor is connected on the rectifier output\& a dc is obtained through it. The filtered waveform is largely a $\mathrm{dc}$ voltage with negligible ripples and it is in the end fed to the load.

\section{H.REGULATORS}

The output voltage from the capacitor is extra filtered \& subsequently regulated. The voltage regulator is a device, which keeps the output voltage steady irrespective of the change in supply variations, load variations \& temperature changes. Here we use fixed voltage regulator specifically LM7805. The IC LM7805 is a $+5 \mathrm{v}$ regulator that is used for microcontroller.

\section{I.BUZZER}

A buzzer or beeper is an audio signaling device, which can be mechanical, electromechanical, or piezoelectric (piezo for short). Typical uses of buzzers and beepers consist of alarm devices, timers, and confirmation of user input which includes a mouse click or keystroke.

\section{TECHNOLOGY USED}

\section{ZIGBEE}

ZigBee is a wireless technology advanced as an open international standard to deal with the unique needs of low-cost, low-strength wireless M2M networks. The ZigBee standard operates at the IEEE 802.15.4 physical radio specification and operates in unlicensed bands such as $2.4 \mathrm{GHz}, 900 \mathrm{MHz}$ and $868 \mathrm{MHz}$ The 802.15.4 specification upon which the ZigBee stack operates, received ratification by the Institute of Electrical and Electronics Engineers (IEEE) in 2003. The specification is a packet-based radio protocol supposed for low-cost, battery-operated gadgets. The protocol lets in gadgets to communicate in a sort of network topologies and may have battery life lasting numerous years.

\section{GSM}

Global System for Mobile (GSM) is a second era cellular standard evolved to cater voice services and information transfer using virtual modulation. GSM is mixture of TDMA (Time Division Multiple Access), FDMA (Frequency Division Multiple Access) and Frequency hopping. Initially, GSM use frequency bands of $25 \mathrm{MHz}$ width : 890 to $915 \mathrm{MHz}$ frequency band for up-link and 935 to $960 \mathrm{MHz}$ frequency for down-link. Later on, two $75 \mathrm{MHz}$ band have been added. 


\section{UART Communication}

Some early telegraph schemes used variable-length pulses (as in Morse code) and rotating clock work mechanisms to transmit alphabetic characters. The first UART like gadgets (with fixed-length pulses) had been rotating mechanical switches (commutators). Various character codes that uses 5, 6, 7, or 8 information bits have become common in teleprinters and later as computer peripherals. Gordon Bell designed the UART for the PDP collection of computer systems. The teletypewriter made an extraordinary general-purpose I/O tool for a small computer. To lessen costs, which includes wiring and back-plane costs, these computer systems additionally pioneered flow control that uses XON and XOFF characters rather than hardware wires.

A well-known asynchronous receiver/transmitter, abbreviated UART is a bit of computer hardware that interprets information between parallel and serial forms. UART are usually used along with communication requirements such as EIA, RS-232, RS-422 or RS-485. The well-known designation suggests that the information format and transmission speeds are configurable. The electric signaling stages and methods (such as differential signaling etc.) are dealt with by a driver circuit outside to the UART.

Communication may be simplex (in a single path only, without a provision for the receiving device to send records again to the transmitting device), full duplex (each gadgets send and receive on the identical time) or half duplex (gadgets take turns transmitting and receiving).

\section{RESULTS AND DISCUSSION}

\section{MENU PROJECTION}

Menu objects will be displayed on the dining table using LCD screen. Customer will pick out what they need from the menu listed, through touching the item display. This project makes use of hologram to display the menu on LCD screen. Fig 6.1 shows the menu items being displayed.

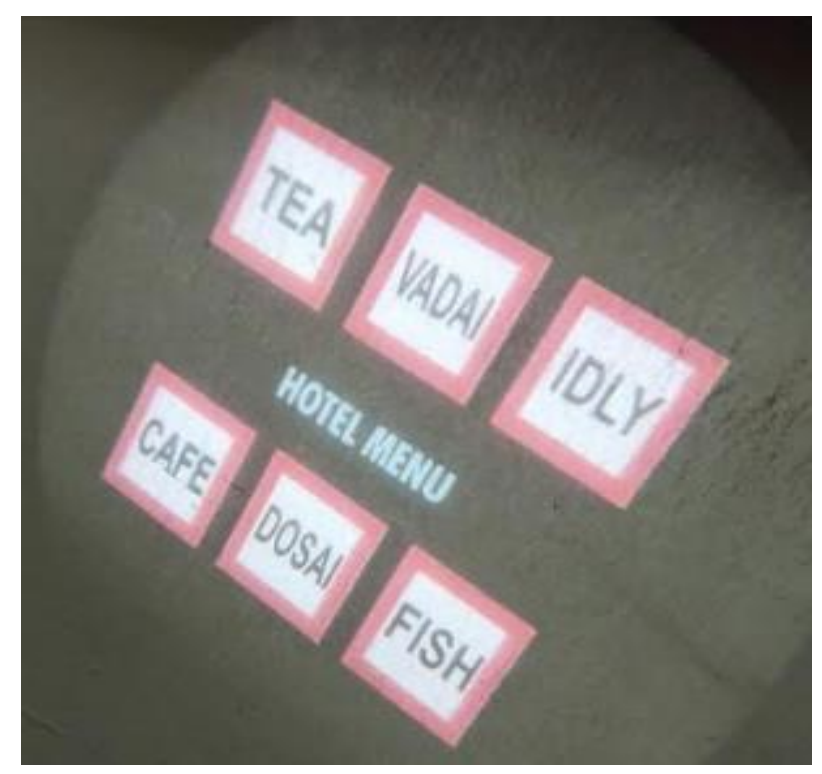

Fig 6.1 Menu Display

\section{TRANSMISSION AND RECEIVING OF MENU ITEMS}

The selected menu item will be transmitted via ZIGBEE, which may be acquired and considered by the chef. Then the chef will begin the preparation as in step with the customer's need.The Transmitter segment is used for the ordering from the menu card that is shown at the LCD display. The primary use of the receiver is to acquire the facts from the transmitter segment. The LCD module is utilized in showing the acquired information. A buzzer is likewise used to make a sound each time a new order is placed in the restaurant. Fig 7.2 shows a user making selection of a menu item. 


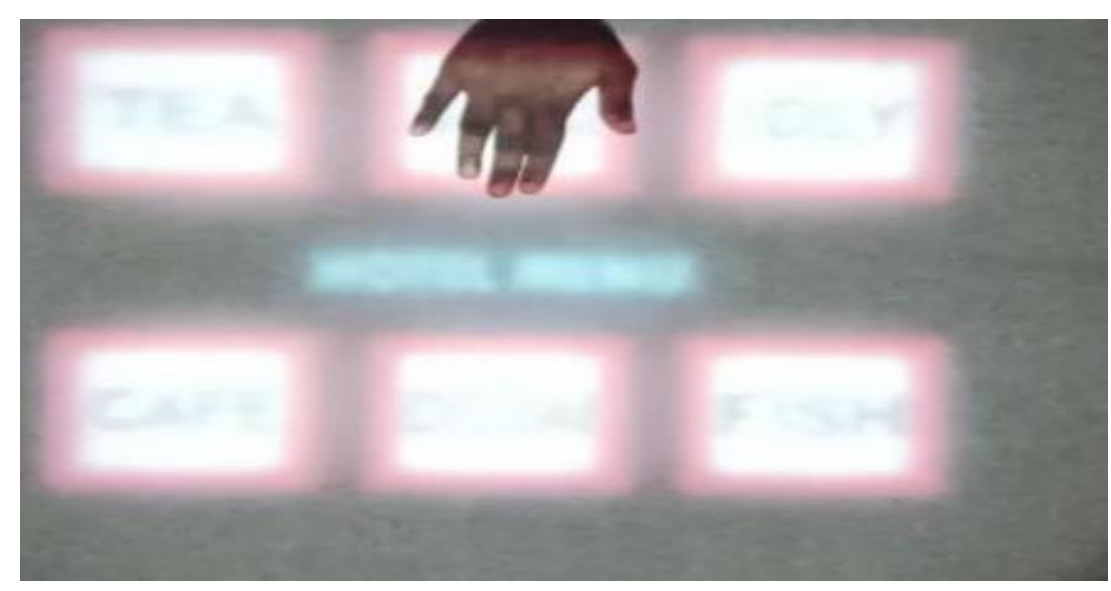

Fig 6.2 Sensing the Menu Item

\section{BILL GENERATION}

The invoice amount for the requested meal is displayed as a message using GSM by clicking a button.

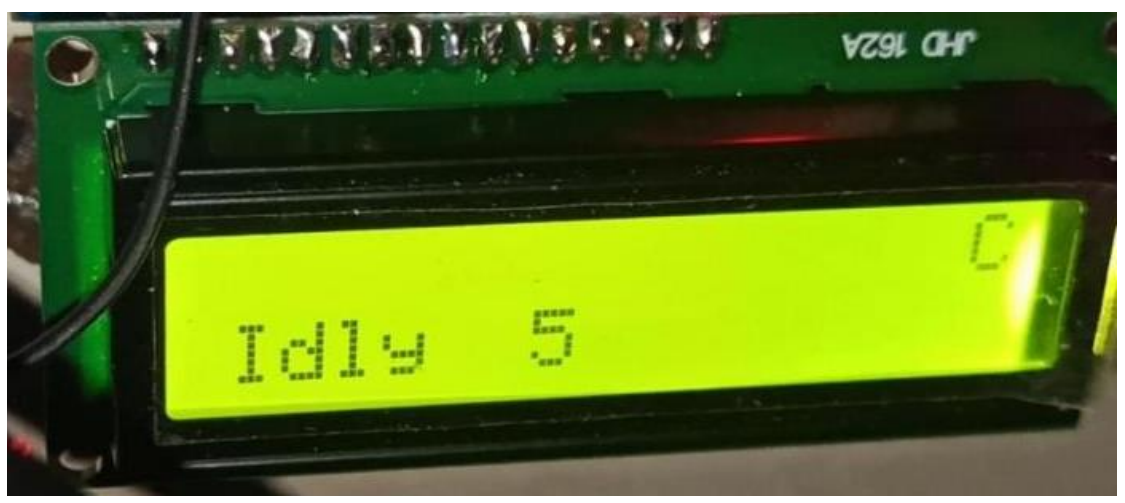

Fig 6.3 LCD Display showing the price of an item

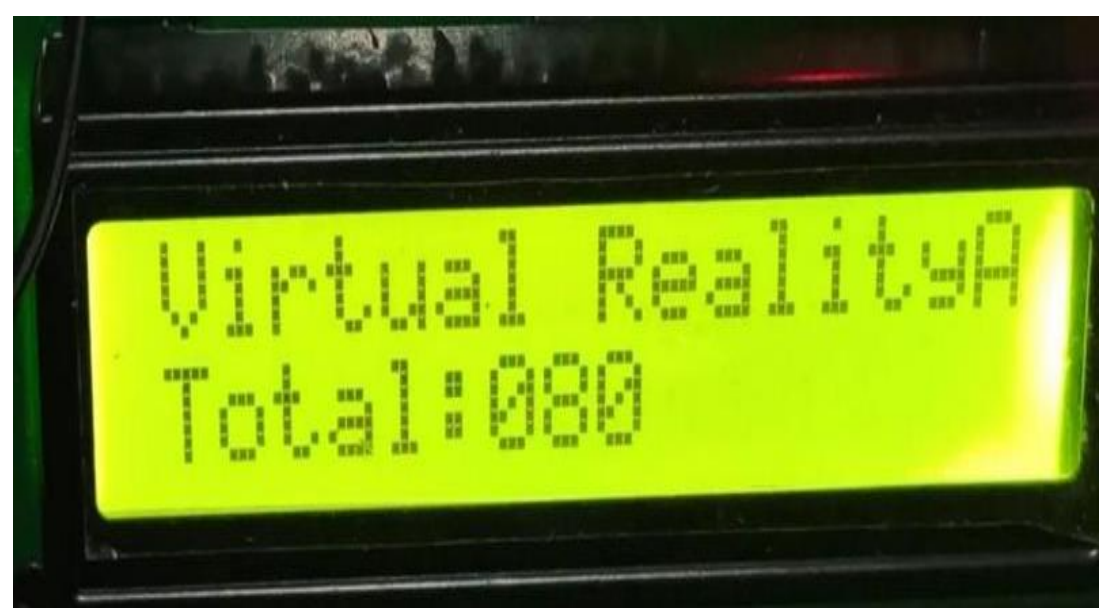

Fig 6.4 LCD display showing the total bill 


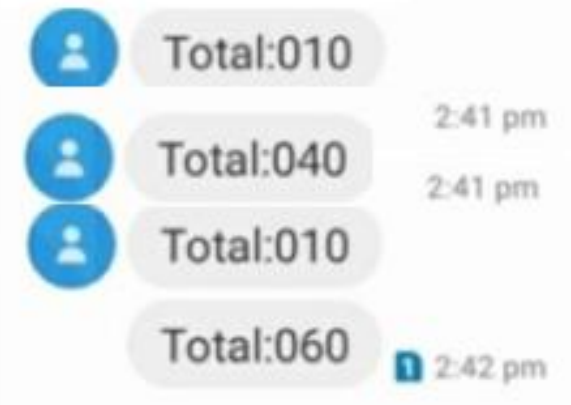

Fig 6.5 Message Received by the Customer

\section{CONCLUSION AND FUTURE ENHANCEMENT}

Our paper implemented the model within a framework that may be used for redirect meals distribution within specific virtual and physical environments. It is beneficial for the assessment of redirected parameters under varying conditions. In this paper, the usage of digital reality food items are displayed via the Buttons like Tea, Dosa, Idly Etc.. In future, long distance display may be achieved via laser beam light.

This project may be utilized in the time of emergency, as a primary useful resource tool. Since the charge of our project is low cost, it is straightforward to buy for all the people. Customers just need some fundamental understanding to operate. In future, payment additionally may be made electronically through growing appropriate modules.

\section{REFERENCES}

[1]. A. McGregor et al., "A Cloud-Based Platform for Supporting Research Collaboration," 2015 IEEE 8th International Conference on Cloud Computing, New York City, NY, 2015, pp. 1107-1110, 2015.

[2]. L. Trappeniers, M. A. Feki, F. Kawsar and M. Boussard. "The internet of things: the next technological revolution." Computer, vol. 46, no. 2, pp. 24-25, 2013

[3]. K. Kamarudin, et al., "The Application of Wireless Food Ordering System," MASAUM Journal of Computing, vol. 1, pp. 178-184, 2009.

[4]. Bastug E, Bennis M, Medard M, Debbah M. Toward interconnected virtual reality: opportunities, challenges, and enablers. IEEE Communications Mag. 2017;55:110-7.

[5]. Wakunami K, Hsieh PY, Oi R, Senoh T, Sasaki H, Ichihashi Y, Okui M, Huang YP, Yamamoto K. Projection-type see-through holographic three-dimensional display. Nat Commun. 2016;7:12954.

[6]. Hirayama R, Plasencia DM, Masuda N, Subramanian S. A volumetric display for visual, tactile and audio presentation using acoustic trapping. Nature. 2019;575:320-3.

[7]. Griffiths AD, Herrnsdorf J, Strain MJ, Dawson MD. Scalable visible light communications with a micro-LED array projector and high-speed smartphone camera. Opt Express. 2019;27:15585-94.

[8]. Zhang H, Li L, Mccray DL, Yao D, Yi AY. A microlens array on curved substrates by 3D micro projection and reflow process. Sensorsand Actuators A Physical. 2012;179:242-50.

[9]. Wang Z, Chen RS, Zhang X, Lv GQ, Feng QB, Hu ZA, Ming H, Wang AT. Resolution- enhanced holographic stereogram based on integral imaging using moving array lenslet technique. Appl Phys Lett. 2018;113:221109.

[10]. Li G, Lee D, Jeong Y, Cho J, Lee B. Holographic display for see-through augmented reality using mirror-lens holographic optical element. Opt Lett. 2016;41:2486-9.

[11]. Tom J. Smy, Scott A. Stewart, Shulabh Gupta, "Surface Susceptibility Synthesis of Metasurface Holograms for Creating Electromagnetic Illusions",2020,IEEE Access PP(99):1-1,DOI:10.1109/ACCESS.2020.2995358

[12]. Yang Wu, Jun Wang, Chun Chen, Chan-Juan Liu, "Generation of Phase-Only Fourier Hologram Based on Double Phase Method and Quantization Error Analysis”,2020,IEEE Photonics Journal, PP(99):1-1,DOI:10.1109/JPHOT.2020.3017503

[13]. Roberto Corda, C.Perra, Hologram Domain Data Compression: Performance of Standard Codecs and Image Quality Assessment at Different Distances and Perspectives (2020), IEEE Transactions on Broadcasting.

[14]. Dongyeon Kim, Seungjae Lee, Jaebum Cho, Dukho Lee, Kiseung Bang, Byoungho Lee, Enhancement of Depth Range in LED-based Holographic Near-Eye Display Using Focus Tunable Device, IEEE Explore, DOI:10.1109/ISIE.2019.8781180

[15]. Jonas Schild, Applying Multi-User Virtual Reality to Collaborative Medical Training, 2018 IEEE Conference on Virtual Reality and 3D User Interfaces (VR), DOI:10.1109/VR.2018.8446160. 